\title{
Unusual MRI Findings for a Drug Resistant Depression
}

Halim Abboud* and Georges L Nawfal

Departement of Neurology, Hotel Dieu Hospital, Beirut, Lebanon

\begin{abstract}
Background: The association between intracranial Dural Arteriovenous Fistulas (dAVF) and psychiatric disorders has been reported in a few anecdoctal reports

Case presentation: A case of severe depression with dAVF is presented. The patient with depression showed poor response to antidepressants, and after a severe headache she was investigated and was found to have a dAVF. The DAVF was successfully treated withmcoil embolization. After this treatment, his depression was improved and the quantity of the antidepressants decreased.

Conclusions: The findings in our case suggest a probable association between dAVF and treatment resistant depression caused by cerebral hypoperfusion especially of the left cerebral hemisphere. It may be necessary to evaluate cerebral vessels in patients suffering from depression by using MRA.
\end{abstract}

Keywords: Drug resistant; Hypoperfusion; Embolization

\section{Background}

Intracranial dural arteriovenous fistulas (dAVF) are acquired fistulous connections between meningeal arteries and dural venous sinus or adjacent cortical vein. Patients with dAVFs may be completely asymptomatic. However, symptoms when present, may range from mild symptoms to fatal hemorrhage. The symptoms depend on the location and venous drainage pattern of the dAVF.

We here report a recent case of dAVF presenting with severe resistant depression, which to our knowledge has not been previously reported.

\section{Case Presentation}

A 65-year-old woman presented to our emergency department complaining of recent headache. Her past medical history was a severe resistant depression, with delusions of persecution and feelings of guilt. She was treated with several antidepressants for four years without much benefit. Afterwards, she became poor at ordinary conversation with her family and complained of forgetfulness and difficulty in doing more than one thing at a time.

On admission, neurological examination revealed no deficit. A brain MRI showed a linear hypo intense signal areas on T2weighted MRI suggesting of dilated draining cortical venous congestion after injection of gadolinium mainly in the left cerebral hemisphere without any abnormal signals of recent haemorrhage (Figure 1).

Angiography showed a dural fistula with supply from the middle meningeal artery, superficial temporal artery and posterior meningeal artery, and thrombosis of the superior sagittal sinus with direct cortical venous drainage. Selective embolization was carried out and cortical venous reflux disappeared on the angiogram after the transvenous embolization with coil for dAVF. The patient did not experience any complications during and after these procedures.

One month after dAVF treatment, the patient reported feeling more motivation, and family members reported improvement in the patient's daily activities.

\section{Discussion}

Mood disorders may be associated with global and regional

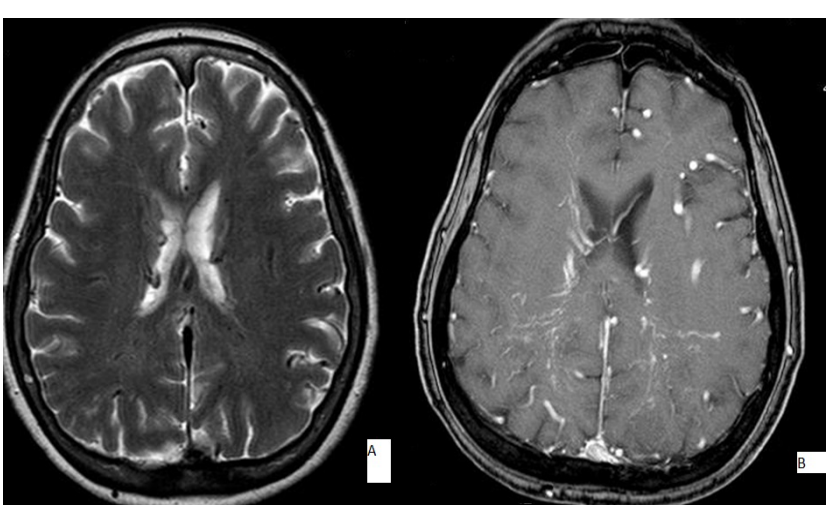

Figure 1: Cerebral MRI showed hypo intense signal areas on T2-weighted MRI (A) and gadolinium enhanced on T1-weighted images suggestion prominent tortuous enhancing venous $(B)$.

changes in cerebral blood. A circuit that connects the limbic system, thalamus, and prefrontal cortex (limbic-thalamic-cortical circuit) was proposed as a neuroanatomical model that plays an important role for the regulation of mood [1]. Reduced perfusion and glucose metabolism in this area may relate to the impairment of cognition accompanying depressive episodes [2].

Dural arteriovenous fistulas are well-known to be associated with brain hemorrhage or venous infarction due to the impediments of the normal venous perfusion. The abundant arterial blood flow via arteriovenous shunts, steno-occlusive changes in the sinuses, and the subsequent abnormal retrograde venous drainage were thought as the main pathological factors. Some patients with dAVFs have been reported to develop the symptoms such as the

*Corresponding author: Halim Abboud, Departement of Neurology, Hotel Dieu Hospital, Beirut, Lebanon, E-mail: halimabboud@hotmail.com

Received December 19, 2014; Accepted December 30, 2014; Published December 31, 2014

Citation: Abboud H, Nawfal GL (2014) Unusual MRI Findings for a Drug Resistant Depression. J Clin Case Rep 4: 468. doi:10.4172/2165-7920.1000468

Copyright: $\odot 2014$ Abboud $\mathrm{H}$, et al. This is an open-access article distributed under the terms of the Creative Commons Attribution License, which permits unrestricted use, distribution, and reproduction in any medium, provided the original author and source are credited. 
Citation: Abboud H, Nawfal GL (2014) Unusual MRI Findings for a Drug Resistant Depression. J Clin Case Rep 4: 468. doi:10.4172/21657920.1000468

progressive dementia, Parkinsonism, transient ischemic attack or other disorders [3-6]. The pathologic areas for these symptoms showed the low perfusion on brain SPECT imaging and improved after proper treatment. Early diagnosis and treatment are important to avoid the development of secondary neurological deficits caused by dAVFs .

The patient responded to the antidepressant therapy after she was treated for the intracranial dural arteriovenous fistulas. This case highlights the role of cerebral perfusion and intracranial venous hypertension in resistant depression.

\section{Conclusions}

Dural arteriovenous fistula is a potentially treatable cause of resistant depression. A high degree of clinical suspicion, detailed clinical examination, and appropriate neuroimaging with cerebral vessels studies are crucial for early diagnosis of this disorder.

\section{References}

1. Price JL, Drevets WC (2010) Neurocircuitry of mood disorders. Neuropsychopharmacology 35: 192-216.

2. Wise T, Cleare AJ, Herane A, Young AH, Arnone D (2014) Diagnostic and therapeutic utility of neuroimaging in depression: an overview. Neuropsychiatr Dis Treat 10: 1509-22.

3. Katz JM, Shetty T, Gobin YP, Segal AZ (2003) Transient aphasia and reversible major depression due to a giant sagittal sinus dural AV fistula. Neurology 557558.

4. Hurst RW, Bagley LJ, Galetta S, Glosser G, Lieberman AP, et al. (1998) Dementia resulting from dural arteriovenous fistulas: the pathologic findings of venous hypertensive encephalopathy. AJNR Am J Neuroradiol 19: 1267-1273.

5. Matsuda S, Waragai M, Shinotoh H, Takahashi N, Takagi K, et al. (1999) Intracranial dural arteriovenous fistula (DAVF) presenting progressive dementia and parkinsonism. J Neurol Sci 165: 43-47.

6. Waragai M, Takeuchi HT, Fukushima T (2006) MRI and SPECT studies of dural arteriovenous fistulas presenting as pure progressive dementia with leukoencephalopathy: a cause of treatable dementia. Eur J Neurol 13: 754-759. 